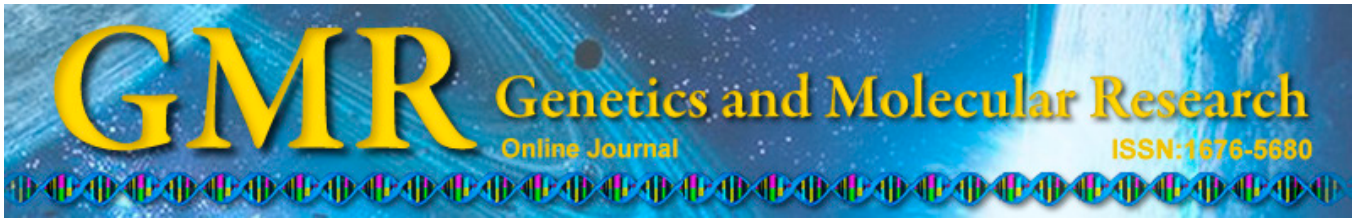

\title{
Protocol to cryopreserve and isolate nuclei from adipose tissue without dimethyl sulfoxide
}

\author{
M.M. Almeida ${ }^{1}$, L.C.J. Caires ${ }^{1}$, C.M. Musso ${ }^{1}$, J.M.S. Campos ${ }^{1}$, \\ C.M.C. Maranduba ${ }^{1}$, G.C. Macedo ${ }^{2}$, J.P.R.F. Mendonça ${ }^{3}$ \\ and R.M.G. Garcia ${ }^{1}$ \\ ${ }^{1}$ Departamento de Biologia, Universidade Federal de Juiz de Fora, \\ Juiz de Fora, MG, Brasil \\ ${ }^{2}$ Departamento de Parasitologia, Microbiologia e Imunologia, \\ Universidade Federal de Juiz de Fora, Juiz de Fora, MG, Brasil \\ ${ }^{3}$ Departmento de Física, Universidade Federal de Juiz de Fora, Juiz de Fora, \\ MG, Brasil \\ Corresponding author: R.M.G. Garcia \\ E-mail: raul.garcia@ufjf.edu.br \\ Genet. Mol. Res. 13 (4): 10921-10933 (2014) \\ Received May 14, 2014 \\ Accepted October 27, 2014 \\ Published December 19, 2014 \\ DOI http://dx.doi.org/10.4238/2014.December.19.14
}

\begin{abstract}
Cryopreservation injuries involve nuclear DNA damage. A protocol for cryopreserving and isolating adipocyte nuclei is proposed. Adipose tissue samples were directly analyzed (NoCRYO$0 \mathrm{~h}$ ), or stored at $-196^{\circ} \mathrm{C}$ for 7 days without $10 \%$ dimethyl sulfoxide (DMSO) (CRYO-WO-DMSO) or with DMSO (CRYO-W-DMSO). To determine the effect of DMSO on cryopreservation treatment, adipose tissue samples were stored at $4^{\circ} \mathrm{C}$ for $24 \mathrm{~h}$ with $10 \% \mathrm{DMSO}$ (NoCRYO-W-DMSO-24h) and without (NoCRYO-WO-DMSO-24h). Samples were processed in isolation buffer, and nuclear integrity was measured by flow cytometry. The coefficient of variation, forward scatter, side scatter, and number of nuclei analyzed were evaluated. Pea (Pisum sativum) was used to measure the amount of DNA. All groups contained similar amounts of DNA to previously reported values and a satisfactory number of nuclei were analyzed. CRYO-W-DMSO
\end{abstract}


presented a higher coefficient of variation $(3.19 \pm 0.09)$ compared to NoCRYO-0h $(1.85 \pm 0.09)$ and CRYO-WO-DMSO (2.02 \pm 0.02$)$. The coefficient of variation was increased in NoCRYO-W-DMSO-24h $(3.80 \pm 0.01)$ compared to NoCRYO-WO-DMSO-24h $(2.46 \pm 0.03)$. These results relate DMSO presence to DNA damage independently of the cryopreservation process. CRYO-W-DMSO showed increased side scatter $(93.46 \pm 5.03)$ compared to NoCRYO-0h $(41.13 \pm 3.19)$ and CRYO-WO-DMSO (48.01 \pm 2.28$)$, indicating that cryopreservation with DMSO caused chromatin condensation and/or nuclear fragmentation. CRYO-W-DMSO and CRYO-WO-DMSO presented lower forward scatter $(186.33 \pm 9.33$ and $196.89 \pm 26.86$, respectively) compared to NoCRYO-Oh $(322.80 \pm 3.36)$, indicating that cryopreservation reduced nuclei size. Thus, a simple method for cryopreservation and isolation of adipocyte nuclei causing less damage to DNA integrity was proposed.

Key words: Adipocyte nuclei; Cryopreservation; Dimethyl sulfoxide; DNA nuclear integrity; Flow cytometry; Isolation

\section{INTRODUCTION}

Cryopreservation techniques involve the cooling of a cell or tissue up to a temperature at which all metabolic processes are arrested (Özkavukcu and Erdemli, 2002). The objective is to use ultra-low temperature to reversibly cease all biological functions of living cells and tissues. In general, cells and tissues are stored at $-196^{\circ} \mathrm{C}$ in liquid nitrogen (Ginani et al., 2012); however, in some cases, $-85^{\circ} \mathrm{C}$ can be used successfully (Woods et al., 2009). Nevertheless, when cryopreservation methods are considered, important factors include not only the storage temperature but also the method of reaching this temperature, as the main cause of cell death is typically not the long-term storage at low temperature, but rather the process from which cells are converted from room temperature to $-60^{\circ} \mathrm{C}$ (Mazur, 1984, 1988). Processes used to reach low temperature can involve slow freezing or ultra-rapid freezing (Khalili et al., 2012). Both procedures may be detrimental to the cells and organelles (Fuller, 2004). The injuries induced by cryopreservation involve many different cell compartments and structures (Stolzing et al., 2012), including nuclear DNA lesions (Riesco and Robles, 2013). Thus, cryoprotectant agents are important because they can be used to improve the survival rate of living cryopreserved cells (Fuller, 2004).

Typical cryoprotectant agents are low-molecular-weight organic compounds that effectively penetrate the cells and prevent intracellular ice crystal formation. These agents include glycerol, dimethyl sulfoxide (DMSO), ethylene glycol, and propylene glycol (Meryman, 2007). DMSO is widely used alone at a $10 \%$ concentration as an effective cryoprotective agent (Pu, 2009). However, at room temperature it is considered toxic (Stolzing et al., 2012).

After cryopreservation treatment, potential damage includes DNA fragmentation, but a number of other types of lesion can occur, such as deletions, creation of abasic sites, base modifications, DNA crosslinks, or epigenetic changes (Riesco and Robles, 2013). However, these results are controversial, likely because of variations in additional factors such as the 
previous state of the sample, the technique used for cryopreservation, or the cryoprotectant applied (Ribas-Maynou et al., 2014). DNA modifications produced by cryopreservation treatment should be examined to ensure the effectiveness of the technique (Riesco and Robles, 2013). Application of cryopreservation to living cells and tissues has revolutionized biotechnology, plant and animal breeding programs, and modern medicine (Fuller, 2004).

A technique for cryopreservation of adipocyte nuclei and their subsequent isolation may be a useful strategy in several research fields. The genetic material of isolated adipocyte nuclei may facilitate measurements to understand gene expression regulation or DNA integrity assessment in various fields. However, it is important that the cryopreservation and isolation methods are not responsible for damaging DNA integrity or modifying their epigenetic profile, which can affect the results obtained after cryopreservation treatment and nuclear isolation. Thus, in this study, we propose a cheaper, safer, and more practical technique for cryopreserving adipocyte nuclei and a simple method for their subsequent isolation to reduce damage to DNA integrity following these processes.

\section{MATERIAL AND METHODS}

\section{Ethics statement}

This study was carried out in strict accordance with the recommendations of the Guide for the Care and Use of Laboratory Animals (http:/grants.nih.gov/grants/olaw/Guide-for-thecare-and-use-of-laboratory-animals.pdf). All procedures involving animals were approved by the Ethics Committee on Animal Experimentation of Universidade Federal de Juiz de Fora at Minas Gerais, Brazil, protocol No. 054/2012. Wistar rats (Rattus norvegicus Berkenhout, 1769), weighing $450-470 \mathrm{~g}$, were kept in a controlled temperature environment $\left(23 \pm 2^{\circ} \mathrm{C}\right)$ with a photoperiod of $12 \mathrm{~h}$ ( 7 a.m. to 7 p.m. light and 7 p.m. to 7 a.m. dark). The animals received water and commercial chow (Nuvital; Colombo, PR, Brazil) ad libitum prior to euthanasia.

\section{Adipose tissue collection and storage}

To determine the effect of DMSO on cryopreserved adipocytes nuclei, the following experiment was performed. Twenty-seven adipose tissue samples were weighed after collection from the retroperitoneal fat of Wistar rats anesthetized with intraperitoneal injection of a $10 \mathrm{mg} / \mathrm{kg}$ xylazine and $90 \mathrm{mg} / \mathrm{kg}$ ketamine solution and euthanized by total exsanguination. Nine adipose tissue samples were immediately processed for nuclear isolation and used for flow cytometry analysis. This group was named No CRYOpreserved and analyzed immediately (NoCRYO-Oh). Nine adipose tissue samples were deposited individually in empty cryotubes; these samples were named CRYOpreserved WithOut DMSO (CRYO-WO-DMSO). Concurrently, nine adipose tissue samples were deposited individually in cryotubes containing $1 \mathrm{~mL} \mathrm{10 \%} \mathrm{DMSO} \mathrm{solution} \mathrm{in}$ fetal bovine serum, and named CRYOpreserved With DMSO (CRYO-W-DMSO). All samples were stored in cryotubes, and then immediately and directly transferred from room temperature $\left(25^{\circ} \mathrm{C}\right)$ to $-196^{\circ} \mathrm{C}$ in liquid nitrogen. After 7 days, all cryotubes were removed from the liquid nitrogen tank, placed directly at room temperature, immediately processed to nuclei isolation, and analyzed using flow cytometry as described below (Figure 1A). 

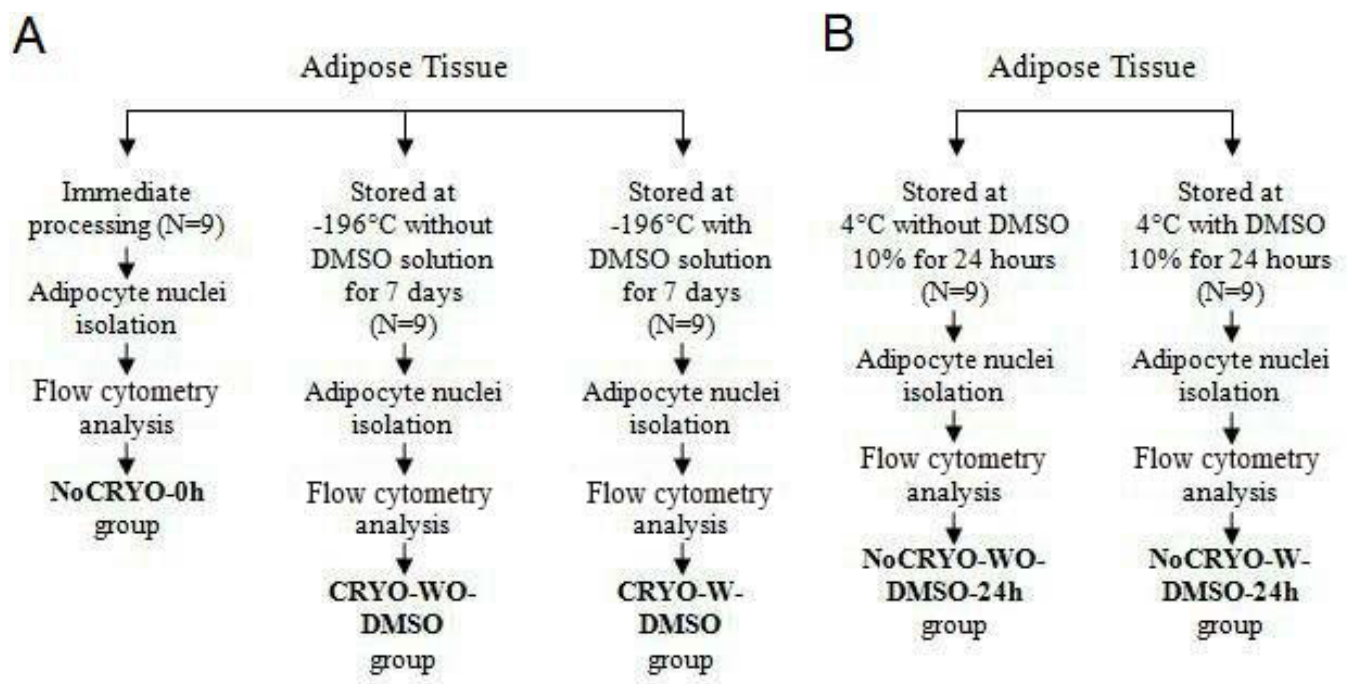

Figure 1. Flowchart showing the experimental design. A. Developed to assess the effect of DMSO on cryopreserved adipocyte nuclei. B. Developed to assess the effect of DMSO on non-cryopreserved adipocyte nuclei.

In order to determine whether the results were affected by cryopreservation in the presence of DMSO or by the presence of DMSO itself, the following experiment was performed. Eighteen adipose tissue samples were weighed after collection from the retroperitoneal fat of Wistar rats as described above. Nine adipose tissue samples were deposited individually in cryotubes containing $1 \mathrm{~mL} \mathrm{10 \%} \mathrm{DMSO} \mathrm{solution} \mathrm{in} \mathrm{fetal} \mathrm{bovine} \mathrm{serum.} \mathrm{This}$ group was named No CRYOpreserved With DMSO for $24 \mathrm{~h}$ (NoCRYO-W-DMSO-24h). Nine adipose tissue samples were deposited individually in cryotubes containing $1 \mathrm{~mL}$ fetal bovine serum. This group was named No CRYOpreserved WithOut DMSO for $24 \mathrm{~h}$ (NoCRYO-WODMSO-24h). All cryotubes were stored at $4^{\circ} \mathrm{C}$ for $24 \mathrm{~h}$. After this period, the samples were placed directly at room temperature, nuclei were isolated, and samples were analyzed by flow cytometry (Figure 1B).

\section{Nuclei isolation of adipose tissue samples}

The nuclei isolation method was adapted from a previous study (Dolezel et al., 2007). Briefly, each adipose tissue sample was individually placed on the center of a plastic Petri dish and $800 \mu \mathrm{L}$ ice-cold nuclei isolation buffer was added. The nuclei isolation buffer composition was $15 \mathrm{mM}$ Tris, $2 \mathrm{mM} \mathrm{Na}$-ethylenediaminetetraacetic acid, $0.5 \mathrm{mM}$ spermine tetrahydrochloride, $80 \mathrm{mM} \mathrm{KCl}, 20 \mathrm{mM} \mathrm{NaCl}$, and $0.1 \%(\mathrm{v} / \mathrm{v})$ Triton X-100, at $\mathrm{pH} 7.5$ adjusted with 1 $\mathrm{M} \mathrm{NaOH}$; the buffer was filtered through a $0.22-\mu \mathrm{m}$ filter and $15 \mathrm{mM} \beta$-mercaptoethanol was added. Adipose tissue samples were immediately chopped in the buffer with a sharp and disposable scalpel. The homogenate was mixed by pipetting several times, avoiding air bubbles, and filtered through a $42-\mu \mathrm{m}$ nylon mesh into a cytometry tube. In this step, we obtained a suspension of isolated adipocyte nuclei for each sample. Finally, to each of the cytometry tubes, we added $50 \mu \mathrm{L}$ propidium iodide. Tubes were individually shaken gently and the nuclear integrity was immediately measured by flow cytometry using the FACSCanto system 
(BD Biosciences; Franklin Lakes, NJ, USA). We evaluated the coefficient of variation (CV), forward scatter (FSC), side scatter (SSC), and number of nuclei analyzed (NNA). A total of 200,000 events/sample were measured. Data were analyzed using the WinMDI 2.8 software.

\section{Determination of DNA amounts}

Three adipose tissue samples were collected from retroperitoneal fat of Wistar rats as described above. Each sample collected was immediately processed in nuclei isolation buffer and analyzed by flow cytometry concurrently with a pea leaf (Pisum sativum), which was used as an internal standard. In addition, flow cytometry analysis of the NoCRYO-0 h, CRYO-WODMSO, and CRYO-W-DMSO groups and the NoCRYO-W-DMSO-24 $\mathrm{h}$ and NoCRYO-WODMSO-24 h groups were always executed together with 3 samples containing only $P$. sativum prepared in nuclei isolation buffer. In all cases, the amount of DNA was determined as follows: mean fluorescence intensity of adipocyte nuclei G1 peak x 9.09 pg (pea DNA amount)/mean fluorescence intensity of pea nuclei G1 peak.

\section{Statistical analysis}

All data are reported as means \pm standard error. Statistical significance $(\mathrm{P}<0.05)$ of experimental observations among the NoCRYO-0h, CRYO-WO-DMSO, and CRYO-W-DMSO groups was determined using the Kruskal-Wallis test followed by Dunn's test. Statistical significance $(\mathrm{P}<0.05)$ of experimental observations between the NoCRYO-W-DMSO-24 $\mathrm{h}$ and NoCRYO-WO-DMSO-24 h groups was determined using the Mann-Whitney test. Analyses were performed using Prism 5.0 (GraphPad Software, Inc.; Palo Alto, CA, USA).

\section{RESULTS}

\section{FLOW CYTOMETRY ANALYSIS OF ADIPOCYTE NUCLEI}

Weights of adipose tissue samples in the NoCRYO-0h, CRYO-WO-DMSO and CRYO-W-DMSO groups were $0.177 \pm 0.012 \mathrm{~g}, 0.231 \pm 0.016 \mathrm{~g}$, and $0.265 \pm 0.022 \mathrm{~g}$, respectively. There were no significant differences among these values. Weights of adipose tissue samples in the NoCRYO-WO-DMSO-24 $\mathrm{h}$ and NoCRYO-W-DMSO-24 h groups were 0.205 $\pm 0.020 \mathrm{~g}$ and $0.221 \pm 0.011 \mathrm{~g}$, respectively, both also statistically equal to each other.

Flow cytometry analysis for the NoCRYO-Oh, CRYO-WO-DMSO, and CRYO-WDMSO groups are shown in Table 1 . The CV of CRYO-W-DMSO group was significantly higher than the CVs of the CRYO-WO-DMSO and NoCRYO-0 $\mathrm{h}$ groups. In addition, there was no significant difference between the CVs of the NoCRYO-0 $\mathrm{h}$ and CRYO-WO-DMSO groups (Figure 2A-C). FSC signals for CRYO-WO-DMSO and CRYO-W-DMSO were not significantly different; however, both results were statistically lower than those for NoCRYO-0 $\mathrm{h}$ FSC. In contrast, there was no significant difference between the SSC signal of CRYO-WODMSO and NoCRYO-0 h groups. However, the SSC of CRYO-W-DMSO was statistically higher than that of CRYO-WO-DMSO and NoCRYO-0 h (Figure 2D-F). For NNA, there was no significant difference between the NoCRYO- $0 \mathrm{~h}$ and CRYO-W-DMSO groups. In addition, the CRYO-WO-DMSO NNA was similar to the CRYO-W-DMSO NNA and was significantly higher than the No-CRYO-0 h NNA. 
Table 1. Flow cytometry analysis of adipocyte nuclei from the retroperitoneal fat of Wistar rats.

\begin{tabular}{lccr}
\hline Parameter & \multicolumn{3}{c}{ Group } \\
\cline { 2 - 4 } & NoCRYO-0 $\mathrm{h}$ & CRYO-WO-DMSO & CRYO-W-DMSO \\
\hline CV $^{1}$ & $1.85 \pm 0.0 \%^{\mathrm{a}}$ & $2.02 \pm 0.02 \%^{\mathrm{a}}$ & $3.19 \pm 0.0 \%^{\mathrm{b}}$ \\
FSC $^{2}$ & $322.80 \pm 3.36^{\mathrm{a}}$ & $196.89 \pm 26.86^{\mathrm{b}}$ & $186.33 \pm 9.33^{\mathrm{b}}$ \\
SSC $^{3}$ & $41.13 \pm 3.19^{\mathrm{a}}$ & $48.01 \pm 2.28^{\mathrm{a}}$ & $93.46 \pm 5.03^{\mathrm{b}}$ \\
NNA $^{4}$ & $806 \pm 60^{\mathrm{a}}$ & $4457 \pm 1244^{\mathrm{b}}$ & $2019 \pm 462^{\mathrm{ab}}$ \\
\hline
\end{tabular}

Adipose tissue samples were collected, processed immediately with the nuclei isolation buffer and analyzed by flow cytometry (NoCRYO-0 h) $\left(\mathrm{N}=9\right.$ ) or collected, stored at $-196^{\circ} \mathrm{C}$ without $10 \%$ DMSO solution (CRYO-WODMSO) $(\mathrm{N}=9)$ or with DMSO (CRYO-W-DMSO) $(\mathrm{N}=9)$ for 7 days, processed with the nuclei isolation buffer, and analyzed. All data are reported as means \pm SE. Statistically significant differences were determined by KruskalWallis test followed by the Dunn test $(\mathrm{P}<0.05)$. Different letters indicate significant difference. ${ }^{1}$ Coefficient of variation; ${ }^{2}$ Forward scatter; ${ }^{3}$ Side scatter; ${ }^{4}$ Number of nuclei analyzed.
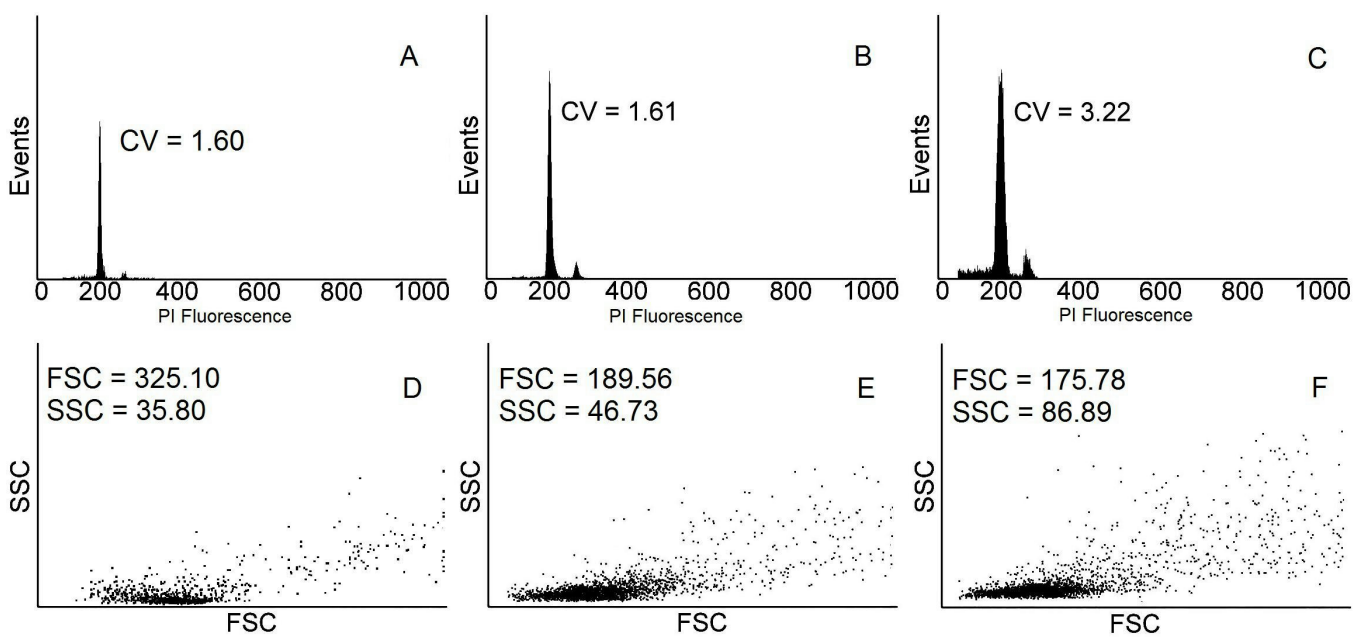

Figure 2. Representative results of flow cytometry analysis of isolated adipocyte nuclei from the retroperitoneal fat of Wistar rats. Adipose tissue samples were collected, processed immediately in nuclei isolation buffer, and analyzed by flow cytometry (NoCRYO- $0 \mathrm{~h})(\mathrm{N}=9)$ or collected, stored at $-196^{\circ} \mathrm{C}$ without $10 \%$ DMSO solution (CRYO-WO-DMSO) $(\mathrm{N}=9)$ or with DMSO (CRYO-W-DMSO) $(\mathrm{N}=9)$ for 7 days, processed with the nuclei isolation buffer, and analyzed. The fluorochrome propidium iodide was used. (A) and (D) NoCRYO-0 h sample; (B) and (E) CRYO-WO-DMSO sample; (C) and (F) CRYO-W-DMSO sample. CV = coefficient of variation; FSC = forward scatter; $\mathrm{SSC}=$ side scatter.

Flow cytometry analysis results for NoCRYO-W-DMSO-24 h and NoCRYO-WODMSO-24 $\mathrm{h}$ are shown in Table 2 . The CV of NoCRYO-W-DMSO-24 h was significantly higher than the CV of NoCRYO-WO-DMSO-24 h (Figure 3A and B). FSC signals for NoCRYO-W-DMSO-24 $\mathrm{h}$ and NoCRYO-WO-DMSO-24 $\mathrm{h}$ were not significantly different. There was no significant difference between the SSC of the NoCRYO-W-DMSO-24 h and NoCRYO-WO-DMSO-24 h groups (Figure $3 \mathrm{C}$ and D). According to the NNA for each sample, there was no significant change between the two experimental groups. 
Table 2. Flow cytometry analysis of non-cryopreserved adipocyte nuclei from the retroperitoneal fat of Wistar rats stored at $4^{\circ} \mathrm{C}$ for $24 \mathrm{~h}$.

\begin{tabular}{lcc}
\hline Parameter & & Group \\
\cline { 2 - 3 } & NoCRYO-WO-DMSO-24 h & NoCRYO-W-DMSO-24 h \\
\hline CV $^{1}$ & $2.46 \pm 0.03 \%^{\mathrm{a}}$ & $3.80 \pm 0.0 \%^{\mathrm{b}}$ \\
FSC $^{2}$ & $295.32 \pm 2.45^{\mathrm{a}}$ & $318.76 \pm 8.93^{\mathrm{a}}$ \\
SSC $^{3}$ & $56.43 \pm 1.82^{\mathrm{a}}$ & $55.91 \pm 3.75^{\mathrm{a}}$ \\
NNA $^{4}$ & $4492 \pm 616^{\mathrm{a}}$ & $3664 \pm 605^{\mathrm{a}}$ \\
\hline
\end{tabular}

Adipose tissue samples were collected, stored at $4{ }^{\circ} \mathrm{C}$ for $24 \mathrm{~h}$ with fetal bovine serum (NoCRYO-WO-DMSO-24 h) $(\mathrm{N}=9)$ and with $10 \%$ DMSO in fetal bovine serum (NoCRYO-W-DMSO-24 h) $(\mathrm{N}=9)$, processed with the nuclei isolation buffer, and analyzed by flow cytometry. All data are reported as means values $\pm \mathrm{SE}$. Statistically significant differences were determined using the Mann Whitney test $(\mathrm{P}<0.05)$. Different letters indicate significant difference. ${ }^{1}$ Coefficient of variation; ${ }^{2}$ Forward scatter; ${ }^{3}$ Side scatter; ${ }^{4}$ Number of nuclei analyzed.
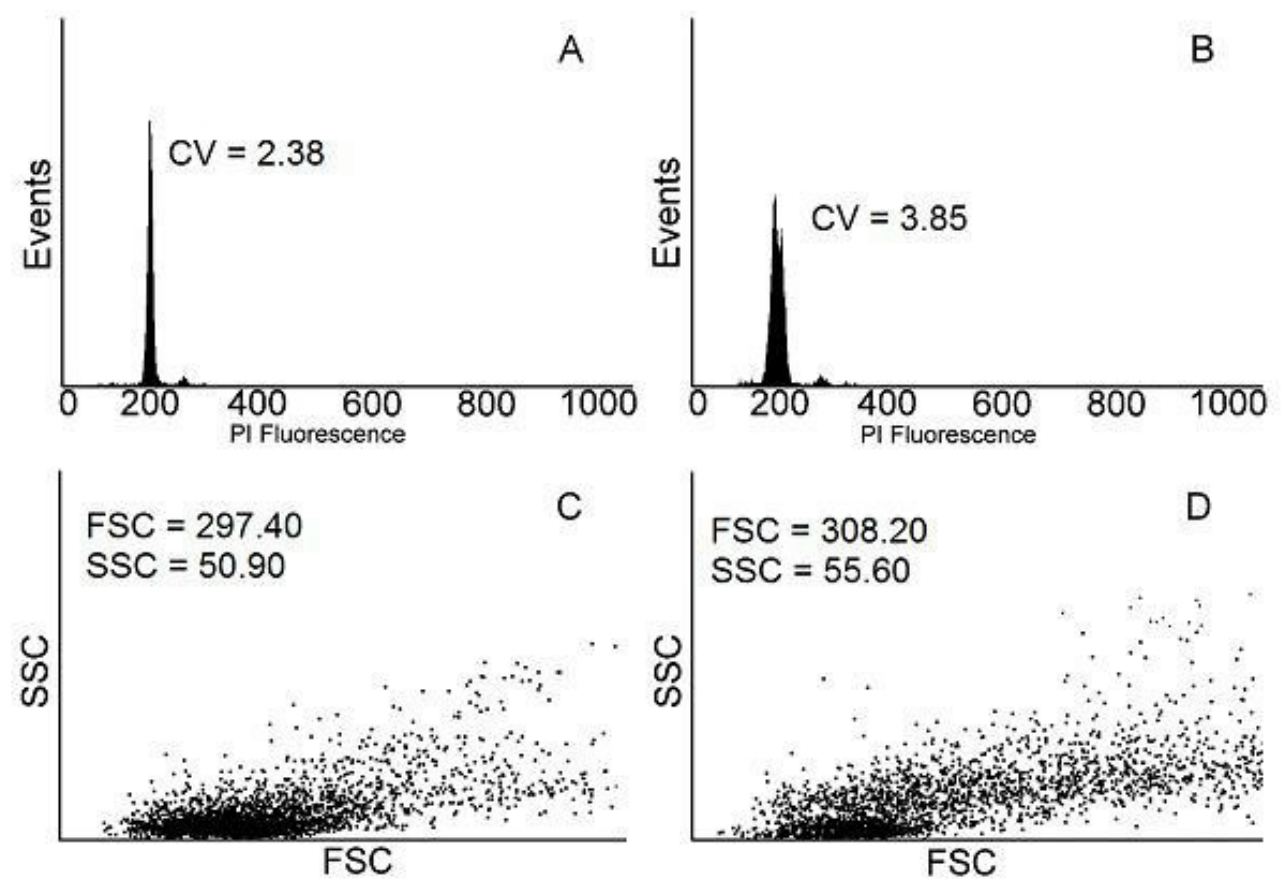

Figure 3. Representative results of flow cytometry analysis of isolated adipocyte nuclei from the retroperitoneal fat of Wistar rats. Adipose tissue samples were collected, stored at $4^{\circ} \mathrm{C}$ for $24 \mathrm{~h}$ with fetal bovine serum (NoCRYOWO-DMSO-24 h) $(\mathrm{N}=9$ ) or with 10\% DMSO in fetal bovine (NoCRYO-W-DMSO-24 h) $(\mathrm{N}=9)$, processed with the nuclei isolation buffer, and analyzed. The fluorochrome propidium iodide was used. (A) and (C) NoCRYOWO-DMSO-24 h sample; (B) and (D) NoCRYO-W-DMSO-24 h sample. CV = coefficient of variation; FSC = forward scatter; $\mathrm{SSC}=$ side scatter.

\section{DNA amount}

The 2C amount of DNA to $R$. norvegicus measured with $P$. sativum as an internal standard was $6.76 \pm 0.10 \mathrm{pg}$ (Figure 4). The 2C amounts of DNA for NoCRYO-0 h, CRYOWO-DMSO, and CRYO-W-DMSO were $6.92 \pm 0.01,6.46 \pm 0.05$, and $6.69 \pm 0.09 \mathrm{pg}$, respec- 
tively. The 2C DNA amounts for NoCRYO-W-DMSO-24h and NoCRYO-WO-DMSO-24 h were $6.74 \pm 0.01$ and $6.59 \pm 0.01 \mathrm{pg}$, respectively.

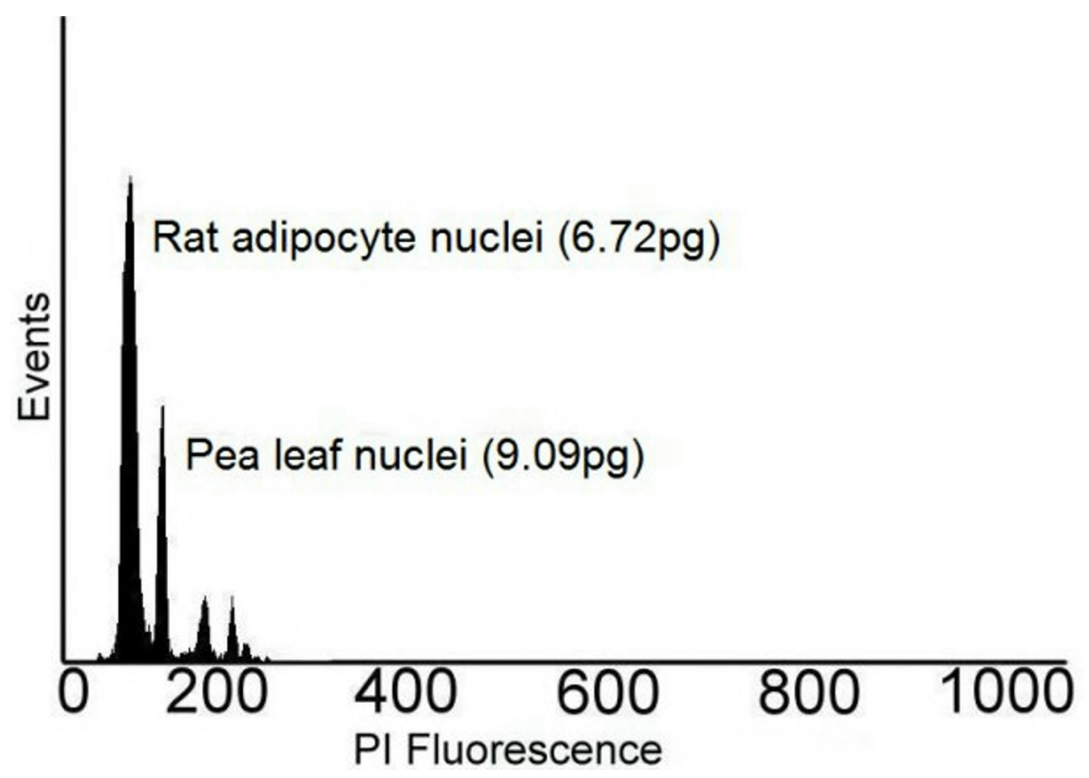

Figure 4. Histogram representing the measurement of DNA amount for rat (Rattus norvegicus) using pea (Pisum sativum) as an internal standard. Adipose tissue samples were collected from the Wistar rat retroperitoneal fat, adipocyte nuclei were immediately isolated together with $P$. sativum nuclei using isolation buffer, and analyzed by flow cytometry. The fluorochrome propidium iodide (PI) was used. The DNA amount was determined as follows: mean fluorescence intensity of adipocyte nuclei G1 peak x $9.09 \mathrm{pg}$ (pea DNA amount)/mean fluorescence intensity of pea nuclei G1 peak.

\section{DISCUSSION}

For effective cryopreservation, the physicochemical characteristics of the specific cell type must be taken into account when predicting the response to freezing (Özkavukcu and Erdemli, 2002). Thus, it is relevant to consider the composition of adipose tissue. Adipocytes from white adipose tissue are characterized morphologically as having a unilocular lipid droplet that occupies $95 \%$ of the cell volume and determines the cell size, which varies from 20 to $200 \mu \mathrm{m}$ (Lee et al., 2013). More than $95 \%$ of the total lipids stored within adipocytes are triglycerides (Lee et al., 2013), and the fat fraction range for white adipose tissue is $90-93 \%$ (Hu et al., 2010). According to a previous study, adipose depots cells were characterized by high lipid and low water contents, with values of approximately $90 \%$ and $10 \%$, respectively (Yang et al., 2013). The low water content in adipose tissue appears to be indispensable for preserving the nuclear integrity of the adipose tissue samples stored without a cryoprotectant agent. This hypothesis is supported by the observation that water concentration in tissues or cells is one of the main factors affecting physical changes during the cooling and warming processes, as water content influences ice crystal formation, which can significantly damage tissues and cells (Stolzing et al., 2012).

Water exhibits anomalous behavior between $4^{\circ} \mathrm{C}$ and $0^{\circ} \mathrm{C}$. In this temperature in- 
terval, it expands rather than contracting (Osamu and Stanley, 1988). However, this volume increase is not sufficient to disrupt the cell membrane. As an example, a cell with a $20-\mu \mathrm{m}$ radius has a volume of $3.45 \times 10^{4} \mu \mathrm{m}^{3}$. Water expansion between $4^{\circ} \mathrm{C}$ and $0^{\circ} \mathrm{C}$ is on the order of $2 \%$; therefore, the new volume and radius may be $3.52 \times 10^{4} \mu \mathrm{m}^{3}$ and $20.3 \mu \mathrm{m}$, respectively. The elasticity of the membrane is extremely high (Diz-Muñoz et al., 2013), supporting the $0.3-\mu \mathrm{m}$ increase in radius. Thus, the temperature decrease until $0^{\circ} \mathrm{C}$ can no longer damage the cell structure.

The critical moment is when the temperature achieves values below $0^{\circ} \mathrm{C}$, when there is formation of ice crystals, which is an under-recognized phenomenon because water nucleation is not well understood (Morris and Acton, 2013). When a large amount of water in the cell cytoplasm is subjected to a rapid temperature decrease to temperatures well below $0^{\circ} \mathrm{C}$, immediate formation of ice crystals occurs in the entire cell volume containing water. Ice crystal formation depends on the presence of water molecules for ice nucleation. Many of these crystals that form have tips, which, as they grow, can perforate the cell membrane and damage cellular compartments. However, ice crystals need to establish an action-reaction pair to exert a force against the cell's structure. If the water content in the cytoplasm is low, the action-reaction pair is not established, and when ice crystals exert pressure to perforate cell compartments, there may be displacement of the mass center of the system and no damage to the cell occurs.

Water content in adipose tissue was approximately 10\% (Yang et al., 2013); thus, when this water percentage exhibits nucleation, the force required to perforate the nuclei is very small. Although ice crystals were formed, the nuclei were preserved. Importantly, the weights of adipose tissue samples were statistically equal, minimizing the interference of sample mass during the freezing and thawing processes, which may have affected the integrity of adipocyte nuclei. Therefore, similar adipose tissue masses indicate that the effects resulted from adipocyte nuclei treatments with and without DMSO as well as the cryopreservation and isolation protocols used.

$\mathrm{CV}$ values obtained using flow cytometry analysis supported this hypothesis because cryopreserved nuclei, even in the absence of a cryoprotectant agent, showed DNA peaks with low CVs, which occur because of the intact nuclei released from cells (Dolezel et al, 2007). Furthermore, low CV values indicate less heterogeneity in the DNA amount of nuclei released from adipose tissue. Propidium iodide, the fluorochrome used in flow cytometry analysis, intercalates into double-stranded DNA without base-dependent bias, making it suitable for estimating DNA amount (Dolezel et al., 2007). High heterogeneity in DNA amount suggests a lower quality of adipocyte nuclei because it represents higher dispersion in the DNA amount, which may be because of structural and numerical chromosomal aberrations as well as the formation of micronuclei (Otto et al., 1981). It was previously shown that treatment with a mutagen drug increased the $\mathrm{CV}$ in a dose-dependent manner, as measured by flow cytometry (Otto et al., 1981).

Nuclear DNA amounts can be analyzed with high precision with the CV of DNA peaks generally ranging from 1 to 5\% (Dolezel et al., 2007); these values are considered acceptable, whereas CVs below 3\% are considered to be good (Dolezel et al., 2007). Thus, it is possible to characterize both methods of cryopreservation without and with $10 \%$ DMSO as acceptable. The CRYO-WO-DMSO CV was significantly lower than the CRYO-W-DMSO CV and was statistically equal to the NoCRYO-0 h CV, indicating the higher quality of adipocyte nuclei that had been cryopreserved without DMSO as the cryoprotectant agent. 
In the NoCRYO-W-DMSO-24 $\mathrm{h}$ and NoCRYO-WO-DMSO-24 $\mathrm{h}$ groups, the CV was significantly higher in the former than for non-cryopreserved nuclei treated without DMSO. Therefore, considering or not cryopreservation treatment, DMSO presence was always associated with an increased CV, indicating that the presence of DMSO itself increased the heterogeneity in the DNA amount of nuclei released from adipose tissue and, consequently, decreased the quality of adipocyte nuclei. This observation is supported by studies that showed the effect of DMSO on increasing the DNA fragmentation percentage and DNA damage (Filipak et al., 2007). Moreover, only groups treated without DMSO, NoCRYO-0 h, CRYO-WO-DMSO, and NoCRYO-WO-DMSO-24 h, had CV values lower than 3\%, which is considered good (Dolezel et al., 2007).

The amounts of DNA obtained from the NoCRYO-0 h, CRYO-WO-DMSO, and CRYO-W-DMSO groups were very similar to the 2C values of $6.10 \mathrm{pg}$ (Vinogradov, 1988) and $6.72 \mathrm{pg}$ (Peterson et al., 1994), which were also determined using flow cytometry for $R$. norvegicus, supporting that the protocol proposed was not responsible for DNA loss. The amount of DNA obtained from the NoCRYO-W-DMSO-24 $\mathrm{h}$ and NoCRYO-WO-DMSO-24 $\mathrm{h}$ groups was also similar to the literature values, demonstrating that DMSO presence was not responsible for DNA loss.

Analysis of nucleus size based on FSC signals (Wlodkowic et al., 2012) revealed that the size of cryopreserved nuclei was not related to the use of DMSO solution, because in both cryopreservation treatments, the nuclei size was smaller than that observed in NoCRYO-0 $\mathrm{h}$ group. Therefore, the decrease in size of the cryopreserved nuclei was not positively correlated with the presence or absence of DMSO as a cryoprotectant agent. This reduction in nuclei size resulted from the low temperature to which these cell compartments were exposed (Atkinson et al., 2006). The FSC signal was statistically equivalent between NoCRYO-W-DMSO-24 h and NoCRYO-WO-DMSO-24 h groups. In addition, the presence of DMSO did not affect nucleus size.

In contrast, nuclear granularity or complexity was analyzed by measuring the SSC signal (Wlodkowic et al., 2012). We found that SSC was significantly increased in CRYOW-DMSO compared to CRYO-WO-DMSO, whereas CRYO-WO-DMSO SSC was statistically equivalent to NoCRYO-0 h SSC. This indicates increased chromatin condensation and/ or nuclear fragmentation (Wlodkowic et al., 2012) in nuclei cryopreserved with DMSO. This result clearly demonstrates the effect of DMSO on cryopreserved adipocyte nuclei. If this damage was caused by the cryopreservation treatment used, it would also be observed in adipocyte nuclei that had been cryopreserved without DMSO, as the CRYO-WO-DMSO and CRYO-WDMSO groups were subjected to the same cryopreservation conditions. Some studies showed that cryopreservation itself caused significant changes in the DNA methylation pattern (Hao et al., 2002a,b; Kaczmarczyk et al., 2010), while another study showed that DNA methylation was not affected by cryopreservation treatment (Klaver et al., 2012). In this study, cryopreservation treatment itself was not responsible for increasing the SSC signal, as this parameter was increased only in the group cryopreserved in DMSO. Therefore, changes in SSC occurred because of cryopreservation treatment in the presence of DMSO and did not result from the cryopreservation treatment itself. Increased chromatin condensation in cryopreserved nuclei with DMSO agrees with the results of previous studies reporting that DMSO affects the cellular epigenetic profile by inducing DNA hypermethylation (Hao et al., 2002a; Kawai et al., 2010). This may be related to chromatin condensation and thus heterochromatin expansion. Analysis of the results of SSC for non-cryopreserved nuclei treated with and without DMSO showed 
that DMSO itself was not associated with increased chromatin condensation, as the SSC NoCRYO-W-DMSO-24 h group was statistically equivalent to the SSC NoCRYO-WO-DMSO-24 $\mathrm{h}$ group. Thus, increased chromatin condensation resulted from cryopreservation in the presence of DMSO, as the SSC of NoCRYO-W-DMSO-24 h group was not increased compared to that of the NoCRYO-WO-DMSO-24 h group, and the SSC of the CRYO-W-DMSO group was increased compared to those of the CRYO-WO-DMSO and NoCRYO-0 h groups.

Based on the number of nuclei analyzed, there are two potential explanations for these results. The first and the most important is related to the nuclei isolation method. It was very difficult to ensure that all samples in isolation buffer were chopped identically, contributing to higher or lower nuclei release from the adipose tissue. This can also explain the high values of the standard error in this parameter. The second reason is regarding the amount of adipose tissue in each sample. Although these values were not statistically different, they were also not identical, which may have contributed to the differences in this parameter among groups. Considering all experimental groups, the number of nuclei analyzed in each group was a low percentage of the 200,000 events, which occurred due to a methodological choice described as follows. The cytometer was configured to count events over a wide interval of fluorescence intensities. This enabled determination of the amount of background debris produced by each cryopreservation treatment. Based on the NNA results, debris formation was not stimulated by cryopreservation without or with DMSO or by DMSO presence in experiments without cryopreservation treatment, as the number of nuclei analyzed was statistically equivalent between the CRYO-WO-DMSO and CRYO-W-DMSO groups and between the NoCRYO-WDMSO-24 $\mathrm{h}$ and NoCRYO-WO-DMSO-24 h groups.

Surfactants were present in the isolation buffer, and they are a chemical class that can cause DNA damage (Yam et al., 1984). Thus, application of nuclei isolation buffer as proposed in this study was validated by determining the DNA content of non-cryopreserved nuclei using pea as an internal standard. The DNA amount measured in adipocytes $(6.76 \pm 0.10 \mathrm{pg})$ was very similar to the $2 \mathrm{C}$ values of $6.10 \mathrm{pg}$ (Vinogradov, 1998) and $6.72 \mathrm{pg}$ (Peterson et al., 1994 ), both also determined using flow cytometry for $R$. norvegicus. This shows that the nuclei isolation buffer was not responsible for DNA loss and demonstrated that the buffer was effective for isolating adipocyte nuclei.

In this study, we proposed a simple method for cryopreserving adipocyte nuclei without using DMSO as cryoprotectant agent, and their subsequent isolation. The use of this technique is dependent upon the specific circumstances. A useful application of the proposed technique is related to the cryopreservation of adipocyte nuclei followed by a simple method for isolating these nuclei. Isolated adipocyte nuclei can be used in studies examining gene expression regulation with numerous protocols. Thus, it is possible to validate methods to quantify nuclear factor-kB (Napetschnig and Wu, 2013), protein kinase A (Prusov et al., 2013), proteins of the Notch pathway (Fiúza and Arias, 2007), and intracellular receptors (Re et al., 2010) in these isolated nuclei. In addition, isolated nuclei transcriptome studies can be conducted based on RNA synthesis and quantification (Grindberg et al., 2013).

Furthermore, our flow cytometry results revealed more injuries in adipocytes nuclear DNA when DMSO was used, indicating that DMSO should not be used in studies evaluating the structure of cryopreserved nuclear DNA, such as when identifying DNA fragmentation using the TdT dUTP nick-end labeling (TUNEL) technique (Wlodkowic et al., 2012), because the presence of DMSO may cause changes in DNA, affecting the results. In addition, nuclei cryopreserved with DMSO presented a significantly increased SSC signal, indicating 
increased chromatin condensation. This result suggests that, when possible, it is better to use alternative cryoprotectant agents to DMSO in cell culture studies, mainly because increased chromatin condensation can silence genes, thereby modifying cell physiology.

In conclusion, we suggest a protocol for the cryopreservation and isolation of nuclei from adipose tissue from Wistar rat retroperitoneal fat stored using an ultra-rapid freezing technique in liquid nitrogen without $10 \%$ DMSO solution. The absence of DMSO as a cryoprotective agent is advantageous because the presence of DMSO appears to cause nuclear DNA damage of adipocytes. Furthermore, cryopreservation in the presence of DMSO was associated with increased chromatin condensation and/or nuclear fragmentation. Moreover, the absence of DMSO and ultra-rapid freezing makes the proposed technique cheaper, easier, more practical, and safer.

\section{ACKNOWLEDGMENTS}

The authors would like to thank Jacy Gameiro from the Parasitology, Microbiology, and Immunology Department of Universidade Federal de Juiz de Fora for technical support. Research supported by Universidade Federal de Juiz de Fora (UFJF) and Coordenação de Aperfeiçoamento de Pessoal de Nível Superior (Capes).

\section{REFERENCES}

Atkinson D, Morley SA and Hughes RN (2006). From cells to colonies: at what levels of body organization does the 'temperature-size rule' apply? Evol. Dev. 8: 202-214.

Diz-Muñoz A, Fletcher DA and Weiner OD (2013). Use the force: membrane tension as an organizer of cell shape and motility. Trends Cell Biol. 23: 47-53.

Dolezel J, Greilhuber J and Suda J (2007). Estimation of nuclear DNA content in plants using flow cytometry. Nat. Protoc. 2: 2233-2244.

Filipak NF, Zanata SM, Silva de Assis HC, Bussolaro D, et al. (2007). Use of hepatocytes from Hoplias malabaricus to characterize the toxicity of a complex mixture of lipophilic halogenated compounds. Toxicol. In Vitro 21: 706-715.

Fiúza UM and Arias AM (2007). Cell and molecular biology of Notch. J. Endocrinol. 194: 459-474.

Fuller BJ (2004). Cryoprotectants: the essential antifreezes to protect life in the frozen state. Cryo Letters 25: 375-388.

Ginani F, Soares DM and Barboza CAG (2012). Influência de um protocolo de criopreservação no rendimento in vitro de células-tronco derivadas do tecido adiposo. Rev. Bras. Cir. Plást. 27: 359-363.

Grindberg RV, Yee-Greenbaum JL, McConnell MJ, Novotny M, et al. (2013). RNA-sequencing from single nuclei. Proc. Natl. Acad. Sci. U. S. A. 110: 19802-19807.

Hao YJ, You CX and Deng XX (2002a). Effects of cryopreservation on developmental competency, cytological and molecular stability of Citrus callus. Cryo Letters 23: 27-35.

Hao YJ, You CX and Deng XX (2002b). Analysis of ploidy and the patterns of amplified fragment length polymorphism and methylation sensitive amplified polymorphism in strawberry plants recovered from cryopreservation. Cryo Letters 23: 37-46.

Hu HH, Smith DL Jr., Nayak KS, Goran MI, et al. (2010). Identification of brown adipose tissue in mice with fat-water IDEAL-MRI. J. Magn. Reson. Imaging 31: 1195-1202.

Kaczmarczyk A, Houben A, Keller ER and Mette MF (2010). Influence of cryopreservation on the cytosine methylation state of potato genomic NDA. Cryo. Letters 31: 380-391.

Kawai K, Li YS, Song MF and Kasai H (2010). DNA methylation by dimethyl sulfoxide and methionine sulfoxide triggered by hydroxyl radical and implications for epigenetic modifications. Bioorg. Med. Chem. Lett. 20: 260-265.

Khalili MA, Maione M, Palmerini MG, Bianchi S, et al. (2012). Ultrastructure of human mature oocytes after vitrification. Eur. J. Histochem. 56: e38.

Klaver R, Bleiziffer A, Redmann K, Mallidis C, et al. (2012). Routine cryopreservation of spermatozoa is safe-evidence from the DNA methylation pattern of nine spermatozoa genes. J. Assist. Reprod. Genet. 29: 943-950.

Lee MJ, Wu Y and Fried SK (2013). Adipose tissue heterogeneity: implication of depot differences in adipose tissue for 
obesity complications. Mol. Aspects Med. 34: 1-11.

Mazur P (1984). Freezing of living cells: mechanisms and implications. Am. J. Physiol. 247: C125-C142.

Mazur P (1988). Stopping biological time. The freezing of living cells. Ann. N. Y. Acad. Sci. 541: 514-531.

Meryman HT (2007). Cryopreservation of living cells: principles and practice. Transfusion 47: 935-945.

Morris GJ and Acton E (2013). Controlled ice nucleation in cryopreservation-a review. Cryobiology 66: 85-92.

Napetschnig J and Wu H (2013). Molecular basis of NF-kappaB signaling. Annu. Rev. Biophys. 42: 443-468.

Osamu M and Stanley HE (1988). The relationship between liquid, supercooled and glassy water. Nature 396: 329-335.

Otto FJ, Oldiges H, Gohde W and Jain VK (1981). Flow cytometric measurement of nuclear DNA content variations as a potential in vivo mutagenicity test. Cytometry 2: 189-191.

Özkavukcu S and Erdemli E (2002). Cryopreservation: basic knowledge and biophysical effects. J. Ankara Med. School 24: 187-196.

Peterson DG, Stack SM, Healy JL, Donohoe BS, et al. (1994). The relationship between synaptonemal complex length and genome size in four vertebrate classes (Osteicthyes, Reptilia, Aves, Mammalia). Chromosome Res. 2: 153-162.

Prusov AN, Smirnova TA and Kolomijtseva GY (2013). Influence of chromatin structure, antibiotics, and endogenous histone methylation on phosphorylation of histones $\mathrm{H} 1$ and $\mathrm{H} 3$ in the presence of protein kinase $\mathrm{A}$ in rat liver nuclei in vitro. Biochemistry 78: 176-184.

Pu LL (2009). Cryopreservation of adipose tissue. Organogenesis 5: 138-142.

Re M, Pampillo M, Savard M, Dubuc C, et al. (2010). The human gonadotropin releasing hormone type I receptor is a functional intracellular GPCR expressed on the nuclear membrane. PLoS One 5: e11489.

Ribas-Maynou J, Fernandez-Encinas A, Garcia-Peiro A, Prada E, et al. (2014). Human semen cryopreservation: a sperm DNA fragmentation study with alkaline and neutral Comet assay. Andrology 2: 83-87.

Riesco MF and Robles V (2013). Cryopreservation causes genetic and epigenetic changes in zebrafish genital ridges. PLoS One 8: e67614.

Stolzing A, Naaldijk Y, Fedorova V and Sethe S (2012). Hydroxyethylstarch in cryopreservation - mechanisms, benefits and problems. Transfus Apher. Sci. 46: 137-147.

Vinogradov AE (1998). Genome size and GC-percent in vertebrates as determined by flow cytometry: the triangular relationship. Cytometry 31: 100-109.

Wlodkowic D, Skommer J and Darzynkiewicz Z (2012). Cytometry of apoptosis. Historical perspective and new advances. Exp. Oncol. 34: 255-262.

Woods EJ, Perry BC, Hockema JJ, Larson L, et al. (2009). Optimized cryopreservation method for human dental pulpderived stem cells and their tissues of origin for banking and clinical use. Cryobiology 59: 150-157.

Yam J, Booman KA, Broddle W, Geiger L, et al. (1984). Surfactants: a survey of short-term genotoxicity testing. Food Chem. Toxicol. 22: 761-769.

Yang Y, Smith DL, Jr., Hu HH, Zhai G, et al. (2013). Chemical-shift water-fat MRI of white adipose depots: inability to resolve cell size differences. Int. J. Body Compos. Res. 11: 9-16. 